\title{
Role of Blue-green-light Filter in Detecting a Spontaneous Cerebrospinal Fluid Rhinorrhea in an Unusual Site
}

\author{
Natarajan Ramalingam ${ }^{1}$, Nithin P Nair ${ }^{2}$, Sunil K Saxena ${ }^{3}$, Jyotirmay S Hegde ${ }^{4}$
}

\begin{abstract}
Proper identification of the site, size, multiple, and bilateral defects is very important to prevent recurrences after cerebrospinal fluid (CSF) leak repair. The utility of intrathecal fluorescein and a blue-light filter in this has been established before. We are reporting a case of a spontaneous CSF leak in an unusual site, which was identified completely with the help of intrathecal fluorescein and blue-light filter and successfully managed by the endoscopic approach.

Keywords: Blue-light filter, Cerebrospinal fluid, Fluorescein, Rhinorrhea.

Clinical Rhinology An International Journal (2018): 10.5005/jp-journals-10013-1344
\end{abstract}

\section{INTRODUCTION}

Cerebrospinal fluid (CSF) rhinorrhea occurs due to a variety of reasons-mainly due to trauma but may be of spontaneous nature in some cases. Identification and closure of leak are important to prevent further communication and avoidance of meningitis in future. ${ }^{1}$ Multiple sites of leak that are not identified intraoperatively are the major reason for the recurrent or persistent leak. Usage of intrathecal fluorescein and a blue-light filter helps in proper identification of the site that is not visible in the normal white light. We are reporting a case of spontaneous CSF leak in an unusual site, which was identified with the help of fluorescein and blue-light filter and successfully managed by the endoscopic approach.

\section{Case Description}

A 40-year-old lady presented to our center with complaints of spontaneous onset right-side nasal discharge, which was clear and watery in nature, and of insidious onset, continuous and progressive in nature and aggravating on bending forward. She was not able to sniff back the nasal discharge. There was no history of sneezing, trauma, or allergy. The nasal discharge was suspected to be CSF on lab examination. On imaging, CT PNS showed a questionable site of a bony defect at the junction of the posterior ethmoid and sphenoid.

The patient was planned for CSF leak repair under general anesthesia. The fluorescein test dose was given with standard precautions (with crash guard), by injecting $0.1 \mathrm{~mL}$ of $5 \%$ fluorescein intradermally. There was no reaction observed. A lumbar drain was inserted through which $0.2 \mathrm{~mL}$ of $5 \%$ fluorescein dye diluted in 10 $\mathrm{mL}$ CSF was injected over 10 minutes. The patient was positioned with head end low for 1 hour.

The CSF leak was observed from right sphenoethmoidal area by identifying the presence of dye. After infiltration, the agger nasi cell was opened followed by anterior and posterior ethmoidectomy. A $0.5 \times 0.5 \mathrm{~cm}$ fluorescein stained meningocele was visualized with leak over the posterior ethmoid and sphenoid bony wall junction (Fig. 1A). Under a blue-light filter, the area was inspected. Multiple sites of leak were observed on the same site (Fig. 1B).

Meningocele was cauterized using bipolar cautery and the defect was skeletonized all around and a defect measuring

\begin{abstract}
${ }^{1-3}$ Department of ENT, Jawaharlal Institute of Postgraduate Medical Education and Research, Puducherry, India

${ }^{4}$ Department of Otolaryngology, Head and Neck Surgery, Jawaharlal Institute of Postgraduate Medical Education and Research, Puducherry, India
\end{abstract}

Corresponding Author: Natarajan Ramalingam, Department of ENT, Jawaharlal Institute of Postgraduate Medical Education and Research, Puducherry, India, Phone: +91 9442033895, e-mail: rnattu8906@ gmail.com

How to cite this article: Ramalingam N, Nair NP, Saxena SK, et al. Role of Blue-green-light Filter in Detecting a Spontaneous Cerebrospinal Fluid Rhinorrhea in an Unusual Site. Clin Rhinol An Int J 2018;11(2 and 3): 52-54.

Source of support: Nil

Conflict of interest: None

$1.5 \mathrm{~cm} \times 1 \mathrm{~cm}$ was visualized and confirmed using a blue-greenfilter. The leak site was covered initially with fat (Fig. 2A). Using a blue-light filter, the persistent site of leak was observed, which was missed in normal light (Fig. 2B).

The defect was closed in layers using fat, fascia and was sealed and made robust using fibrin glue. The leak site was examined again with a blue-light filter and no sites of leak were observed (Fig. 3). The nasal cavity was packed with $8 \mathrm{~cm}$ Merocel.

The pack was removed followed by lumbar drain on postoperative day 5. The patient was discharged on postoperative day 6 , on oral antibiotics. The patient was followed up regularly for 6 months following which was found to asymptomatic.

\section{Discussion}

Hirsch was the first one, who advocated the transnasal approach for CSF leak repair. ${ }^{2}$ The endoscopic approach was initially documented by Wigand in 1981, which got popularized because of better visualization, rapid recovery, discharge, preservation of olfaction, avoidance of craniotomy scar, and better postoperative results. ${ }^{3}$

The most common cause of CSF rhinorrhea is trauma, which includes $80-90 \%$ of cases. ${ }^{4}$ Among the spontaneous CSF rhinorrhea (nontraumatic), $45 \%$ of cases are due to raised intracranial pressure ${ }^{5}$ 

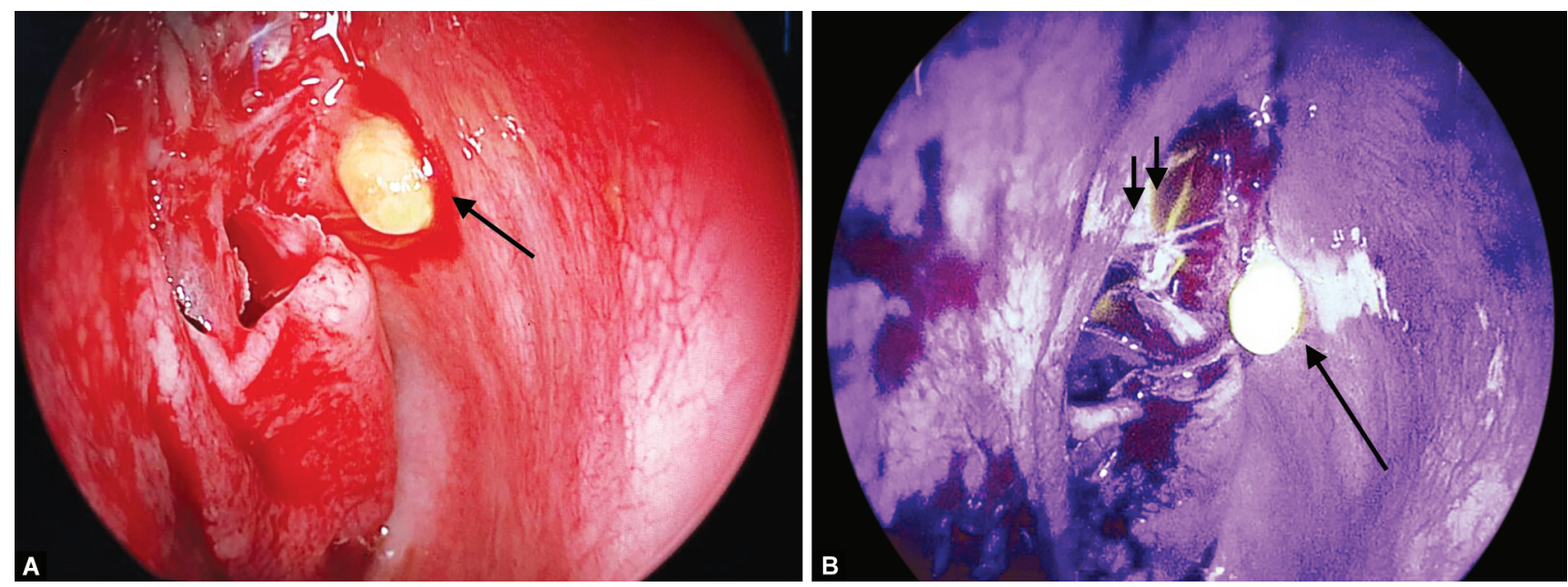

Figs $1 \mathrm{~A}$ and B: (A) Possible site of leak without filter (arrow); (B) Multiple sites of leak with help of a blue-light filter (long and short arrows)
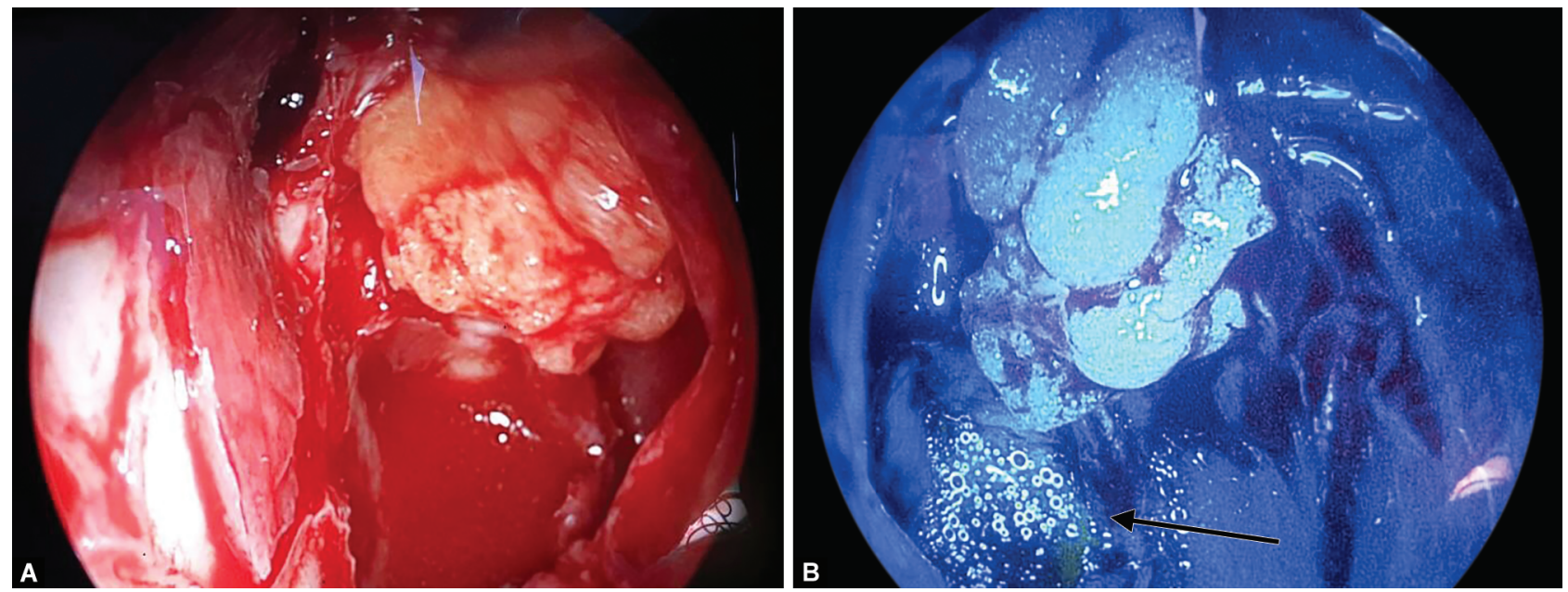

Figs 2A and B: (A) Leak site closed with fascia and fat; (B) Persistent leak (arrow) seen under a blue-light filter
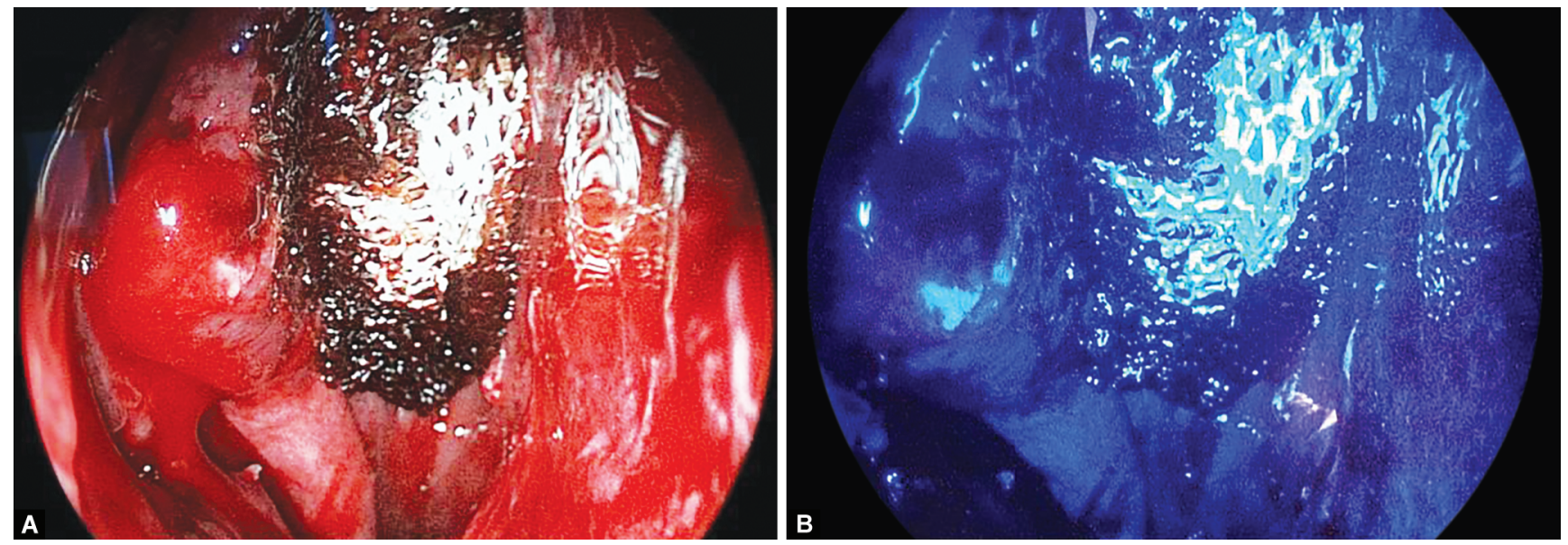

Figs 3A and B: (A) Leak closed in a sandwich manner as described in text; (B) Confirmation of no residual leak with blue-light filter

and $55 \%$ of cases have normal pressure leak. ${ }^{6}$ The spontaneous leak is due to physiological alteration in CSF pressure, which leads to bone erosion. Possible sites of the predilection are the cribriform plate, craniopharyngeal canal, sella, and spheno-occipital synchondrosis area. ${ }^{7}$
Imaging has a huge role in the identification of the leak site. Imaging modalities like computed tomography (CT) cisternography and magnetic resonance (MR) cisternography can aid information regarding the site of the leak and if any meningocele or meningoencephalocele is present. ${ }^{8}$ Even 
though not approved by the FDA, intrathecal fluorescein has been used regularly for intraoperative assessment of the site of leak. ${ }^{9}$

Once fluorescein is diluted to $0.1 \%$, it will emit yellowish light. Fluorescein molecules undergo excitement when exposed to a light of wavelength $460-490 \mathrm{~nm}$, temporarily entering a highenergy state. As fluorescein molecules return to the unexcited stage, absorbed energy is released creating a yellow light of 530-560 nm. Hence, with the help of a blue-light filter the sites of CSF leak will be visualized better, which may be missed when seen without a filter. The filter also helps us in checking whether closure of the leak was adequate and also for checking any residual leaks. ${ }^{9,10}$ The success rate of CSF leak closure is almost $>90 \%$ by the endoscopic approach.

Our case is unique in following things, which include:

- Unusual site of CSF leak, the junction of posterior ethmoid with the sphenoid wall, was noticed in this case.

- The utility of a blue-light filter for identification of the CSF leak site and for identifying any residual leak.

\section{Conclusion}

An unusual site of CSF leak was observed in this patient. With the help of intrathecal fluorescein and a blue-light filter, better visualization of the leak site was done, followed by repair of CSF leak. Any residual leak site can be visualized after the closure of the leak site by the use of this filter along with fluorescein.

\section{References}

1. Sharma D, Singh D, Sinha S, et al. CSF rhinorrhea: an overview of endoscopic repair. Indian J Neurot (IJNT) 2010;7(2):157-162. DOI: 10.1016/S0973-0508(10)80032-2.

2. Yadav YR, Parihar V, Janakiram N, et al. Endoscopic management of cerebrospinal fluid rhinorrhea. Asian J Neurosurg 2016;11(3). DOI: 10.4103/1793-5482.145101.

3. Adams AS, Francis DO, Russell PT. Outcomes of outpatient endoscopic repair of cerebrospinal fluid rhinorrhea. Internation Forum Aller Rhinol 2016(11). DOI: 10.1002/alr.21810.

4. Abuabara A. Cerebrospinal fluid rhinorrhoea: diagnosis and management. Med Oral Patol Oral Cir Bucal 2007;12(5):E397-E400.

5. Schlosser RJ, Woodworth BA, Wilensky EM, et al. Spontaneous cerebrospinal fluid leaks: a variant of benign intracranial hypertension. Ann Otol Rhinol Laryngol 2006;115(7):495-500. DOI: 10.1177/000348940611500703.

6. Lopatin AS, Kapitanov DN, Potapov AA. Endonasal endoscopic repair of spontaneous cerebrospinal fluid leaks. Arch Otolaryngol Head Neck Surg 2003;129(8):859-863. DOI: 10.1001/archotol.129.8.859.

7. Banks CA, Palmer JN, Chiu AG, et al. Endoscopic closure of CSF rhinorrhea: 193 cases over 21 years. Otolaryngol Head Neck Surg 2009;140(6):826-833. DOI: 10.1016/j.otohns.2008.12.060.

8. Lund VJ, Savy L, Lloyd G, et al. Optimum imaging and diagnosis of cerebrospinal fluid rhinorrhoea. J Laryngol Otol 2000;114(12): 988-992. DOI: 10.1258/0022215001904572.

9. Kljajić V, Vuleković , Vlaški L, et al. Endoscopic repair of cerebrospinal fluid rhinorrhea. Braz J Otorhinolaryngol 2016(4). DOI: 10.1016/ j.bjorl.2016.04.024.

10. Singh NP, Roberts DN. An inexpensive blue filter for fluoresceinassisted repair of cerebrospinal fluid rhinorrhea. Laryngoscope 2014;124(5):1103-1105. DOI: 10.1002/lary.24502. 